\title{
Motility-Induced Phase Separation
}

\section{Michael E. Cates ${ }^{1}$ and Julien Tailleur ${ }^{2}$}

${ }^{1}$ SUPA, School of Physics and Astronomy, University of Edinburgh, JCMB Kings Buildings, Mayfield Road, Edinburgh EH9 3JZ, UK; m.e.cates@ed.ac.uk

${ }^{2}$ Univ Paris Diderot, Sorbonne Paris Cité, MSC, UMR 7057 CNRS, F75205

Paris, France; julien.tailleur@univ-paris-diderot.fr
Xxxx. Xxx. Xxx. Xxx. YYYY. 00:1-26

This article's doi:

$10.1146 /(($ please add article doi $))$

Copyright @ YYYY by Annual Reviews. All rights reserved

\section{Keywords}

self-propelled particles, bacteria, phase separation, motility, active Brownian, run-and-tumble

\section{Abstract}

Self-propelled particles include both self-phoretic synthetic colloids and various micro-organisms. By continually consuming energy, they bypass the laws of equilibrium thermodynamics. These laws enforce the Boltzmann distribution in thermal equilibrium: the steady state is then independent of kinetic parameters. In contrast, self-propelled particles tend to accumulate where they move more slowly. They may also slow down at high density, for either biochemical or steric reasons. This creates positive feedback which can lead to motility-induced phase separation (MIPS) between dense and dilute fluid phases. At leading order in gradients, a mapping relates variable-speed, self-propelled particles to passive particles with attractions. This deep link to equilibrium phase separation is confirmed by simulations, but generally breaks down at higher order in gradients: new effects, with no equilibrium counterpart, then emerge. We give a selective overview of the fast-developing field of MIPS, focusing on theory and simulation but including a brief speculative survey of its experimental implications. 


\section{Contents}

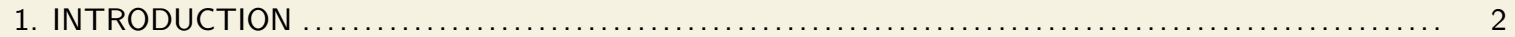

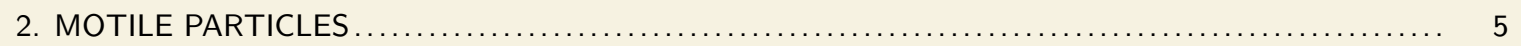

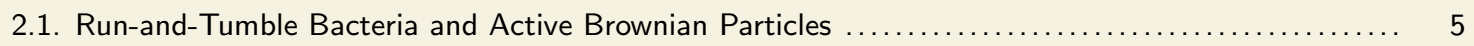

2.2. Spatial Variations in Motility Parameters: a mesoscopic approach $\ldots \ldots \ldots \ldots \ldots \ldots \ldots \ldots \ldots \ldots \ldots .6$

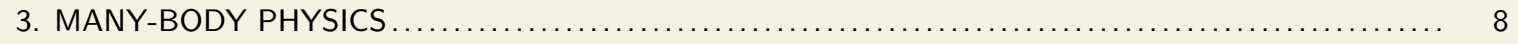

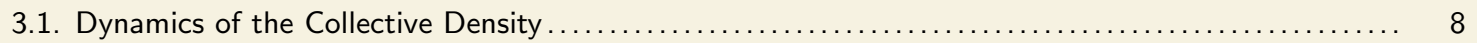

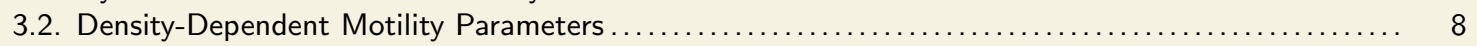

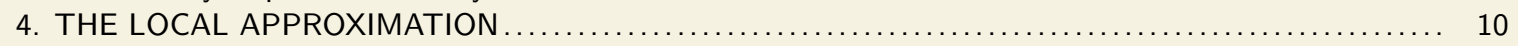

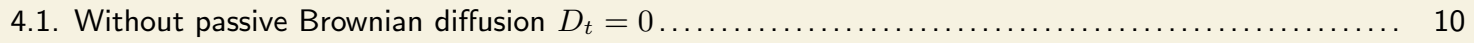

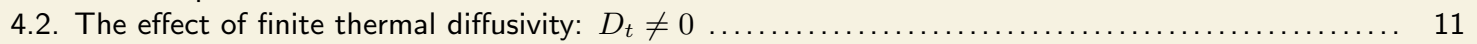

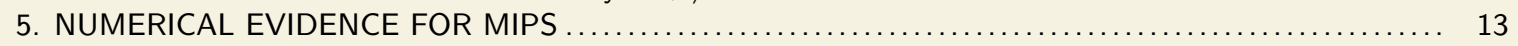

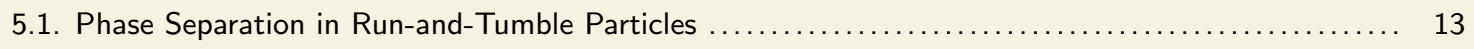

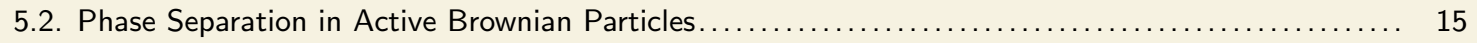

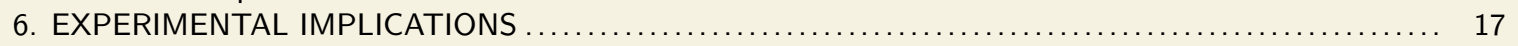

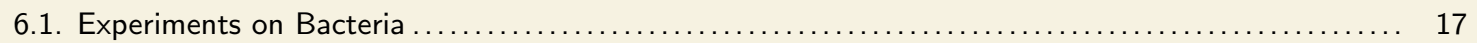

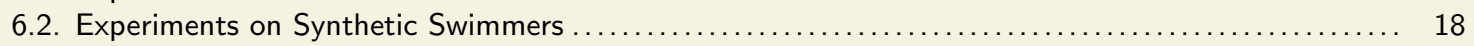

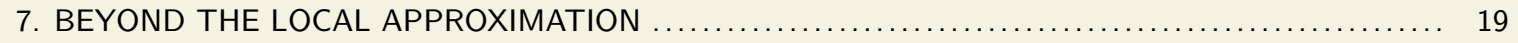

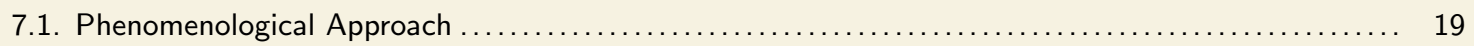

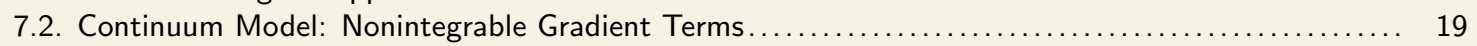

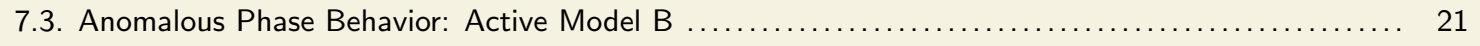

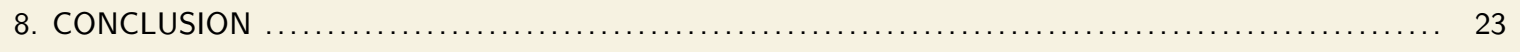

\section{INTRODUCTION}

Non-equilibrium systems arise in a wide range of situations with very different phenomenologies. Nonetheless, one can identify general categories that share sufficient ingredients to form coherent classes. One such class describes systems that are relaxing towards, but have not yet reached, thermal equilibrium. This relaxation may be relatively unhindered, or might become extremely slow (as happens in glasses). Nonetheless, there is a sense of a direction in which the system either moves, or would move if it could. A second class of non-equilibrium systems describes those whose bulk dynamics is prevented from attaining equilibrium by boundary conditions imposing non-zero steady currents. These are exemplified by heat flow experiments, in which a piece of matter is connected to two reservoirs held at different temperatures.

In a third class of non-equilibrium systems, often called 'active matter', energy is dissipated at the microscopic scale in the bulk, so that each constituent of the system has an irreversible dynamics. This includes a large range of systems whose particles are 'motile', i.e., self-propelled: bird flocks (1), fish schools (2), actin filaments (3) and microtubules (4) in motility assays, autophoretic colloids $(5,6,7)$, and "colloidal rollers" (8). Many such systems have been studied in their own right (9), but over the past ten years, the quest for a generic description of active matter has attracted growing interest $(10,11,12)$. It is reasonable to hope that self-propelled particles, which otherwise interact via standard equilibrium forces (attraction, repulsion, alignment, etc.), might form a coherent sub-class of non-equilibrium systems that can be described by a common theoretical framework.

Active matter systems can exhibit many new behaviors, at least some of which should 
prove relevant to applications. For instance, many forms of bacterial contamination (from coronary implants to domestic water systems $(13,14)$ ) arise from biofilm formation on surfaces, whose early stages are triggered when the local population density exceeds some threshold (15). It would therefore be helpful to understand how a nonuniform density can arise from a uniform one. Also, many studies of motile synthetic colloids (for instance (7)) are motivated by a desire to direct the assembly of nanostructures. More generally one would like to extend our control over soft matter systems, whose applications range from liquid crystal displays to cosmetics and food processing industries, to include 'hybrid' materials in which at least some of the components are active. Examples might include tissue scaffolds (16) for wound repair and electrode systems for microbial fuel cells (17).

The study of active matter has historically been driven mainly by work on biological systems. These are, however, often quite complex: for instance, the rich phenomenology observed in dense swarms of bacteria stems in combination from their self-propulsion, the alignment interactions due to their rod-like shapes, and their hydrodynamic coupling to the medium in which they swarm (18). One fruitful line of research, following the seminal work of Vicsek and co-workers (9), has been dedicated to motile particles with orientational order. This spans polar particles (9), active nematics (19), self-propelled rods (20) and active Ising spins (21); the interplay between interaction-induced alignment of motile particles and their self-propulsion has led to the discovery of a variety of new phases (such as the 'zooming bionematic' phase (22)) and various transitions between these phases.

Inspired by the increasing experimental availability of synthetic motile colloids of somewhat simpler geometry, theorists have recently also addressed simpler models in which (because they are dilute or of spherical shape) swimmers have no innate tendency towards orientational order. Even dilute active suspensions can give nontrivial density profiles in sedimentation equilibrium $(23,24,25)$, and their microscopic irreversibility becomes manifest via rectification interactions with mesoscopic ratchets $(26,27,23,28,29,30)$. In these dilute cases interactions between particles can essentially be neglected, showing that the active equivalent of an ideal gas is already a highly non-trivial object of study.

A first step beyond this non-interacting limit is to consider 'active simple fluids' made of spherical self-propelled particles whose interactions are isotropic. The latter not only includes conventional attractive and repulsive interactions of colloidal spheres but also allows for some forms of signaling, such as quorum sensing in bacteria (31), where a chemical species is emitted isotropically by each particle and its concentration detected. (Note that bacteria cannot directly detect vector quantities such as chemical concentration gradients, but do so indirectly by integrating temporal information as they move. This means they lack the long-range orientational interactions of, say, bird flocks, in which individuals visually detect the mean velocity vector of their neighbors.) Even without any orientational interactions, active simple fluids have a phenomenology much richer than their passive counterparts. Most notably, self-propelled particles with purely repulsive interactions can undergo liquid-gas phase separation $(32,33,34,35,36)$. This is impossible for passive colloidal particles without attractions and stems from an intrinsically non-equilibrium mechanism, called Motility-Induced Phase Separation (MIPS) (37), which is the subject of this review. When the speed of motile particles decreases sufficiently steeply as their local density increases, a uniform suspension becomes unstable, leading to a phase-separated state where a dilute active gas coexists with a dense liquid of substantially reduced motility.

Before reviewing this surprisingly generic phenomenon in depth, we summarize its underlying mechanism, which can be intuitively captured by a relatively simple argument. A

MIPS:

Motility-Induced Phase Separation 
first ingredient, carefully explored by Schnitzer for the case of run-and-tumble bacteria (38), is that active particles generically accumulate where they move more slowly. This follows directly from the master equation of a self-propelled particle of spatially varying speed $v(\mathbf{r})$ :

$$
\dot{P}(\mathbf{r}, \mathbf{u})=-\nabla \cdot[v(\mathbf{r}) \mathbf{u} P(\mathbf{r}, \mathbf{u})]+\Theta[P(\mathbf{r}, \mathbf{u})]
$$

Here $\Theta[P]$ accounts for the changes of the particle orientation $\mathbf{u}$. (For instance, $\Theta[P]=$ $D_{r} \Delta_{\mathbf{u}} P$ for Brownian rotational diffusion.) For isotropic processes, $P_{\text {stat }}(\mathbf{r}, \mathbf{u}) \propto 1 / v(\mathbf{r})$ is always a steady-state solution of Eq. 1. This effect is crucially absent for the Brownian motion of particles in thermal equilibrium. In that case, $v$ is a random variable whose statistics are entirely independent of $\mathbf{r}$, instead depending solely on temperature. (This is the equipartition theorem for kinetic energies.) Therefore a similar effect can only arise in a passive system if the temperature is nonuniform $(39,40)$ : although the diffusivity of isothermal Brownian particles might vary with position, for instance due to gradients of viscosity, this has no effect on $P_{\text {stat }}$, which at uniform temperature is a function of energy only.

The second crucial ingredient of MIPS arises in an assembly of active particles exhibiting a propulsion speed $v$ that depends on the local particle density $\rho$. Such a dependence might arise directly by chemical signaling (e.g., quorum sensing (31)) or by coarse-graining a traditional colloidal interaction such as steric exclusion (see Sec. 5.2 below). MIPS arises from the positive feedback between this accumulation-induced slowing and the slowinginduced accumulation implicit in Eq. 1. Heuristically, consider a small perturbation $\delta \rho(\mathbf{r})$ around a uniform profile $\rho_{0} \equiv c / v\left(\rho_{0}\right)$ where $c$ is some constant. This leads to a spatially varying speed $v\left(\rho_{0}+\delta \rho(\mathbf{r})\right)=v\left(\rho_{0}\right)+v^{\prime}\left(\rho_{0}\right) \delta \rho(\mathbf{r})$ so that $\delta \rho(\mathbf{r})$ and $\delta v(\mathbf{r})$ are in antiphase if $v(\rho)$ decreases with $\rho$. The steady state density for this $v(\mathbf{r})$ would be $\rho_{0}+\delta \rho^{\prime}$ where

$$
\rho_{0}+\delta \rho^{\prime}=\frac{c}{v\left(\rho_{0}\right)+v^{\prime}\left(\rho_{0}\right) \delta \rho} \simeq \frac{c}{v\left(\rho_{0}\right)}\left(1-\frac{v^{\prime}\left(\rho_{0}\right)}{v\left(\rho_{0}\right)} \delta \rho\right)=\rho_{0}-\rho_{0} \frac{v^{\prime}\left(\rho_{0}\right)}{v\left(\rho_{0}\right)} \delta \rho
$$

A linear instability is therefore expected whenever $\delta \rho^{\prime}>\delta \rho$, i.e., when

$$
\frac{v^{\prime}\left(\rho_{0}\right)}{v\left(\rho_{0}\right)}<-\frac{1}{\rho_{0}}
$$

As we will show below, Eq. 3 correctly identifies the region where macroscopic MIPS is initiated by spinodal decomposition. One can however go far beyond this simple linear stability analysis. Indeed, in large measure the coarse-grained dynamics of active simple fluids can be mapped onto the equilibrium dynamics of passive simple fluids with attractive interactions. This allows a large body of knowledge on that case to be easily transferred. However, the departures from this mapping are also interesting, since they point to aspects where the underlying non-equilibrium character of MIPS cannot be transformed away.

This underlying character stems directly from the lack of microscopic time-reversal sym-

DB: Detailed Balance metry in active systems, which means that their steady states need not obey the principle of detailed balance (DB). This principle states that if phase space is divided up into regions, the probability flux from region $A$ into region $B$ is the same as the reverse flux. This precludes circulating fluxes in steady state (for instance $A \rightarrow B \rightarrow C \rightarrow A$ ). Such fluxes are commonly seen in boundary driven systems (such as Bénard convection rolls) and can also feature prominently in active matter $(11,23,28,29,30)$. In active matter DB violations are always present microscopically, but may or may not survive coarse-graining. Only when they do not survive, is any mapping possible onto an equivalent equilibrium system. 

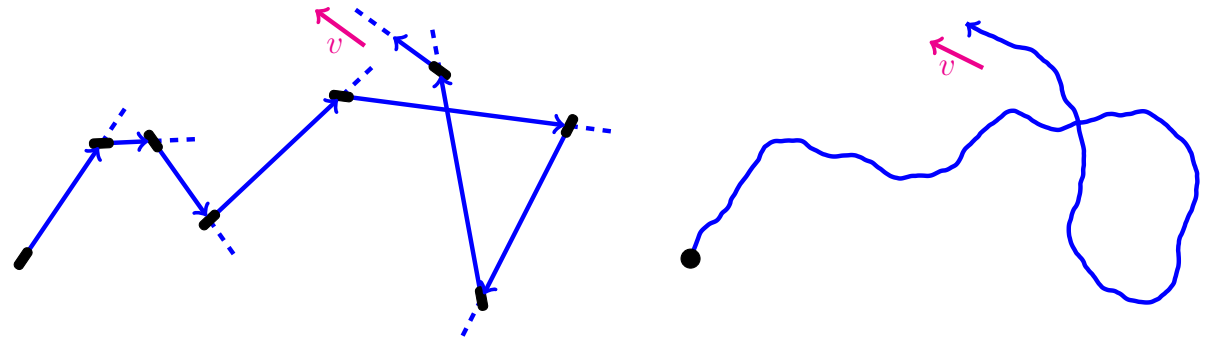

Figure 1

Simulated paths of a run-and-tumble (Left) and an active Brownian (Right) particles of length $\tau=5 \alpha^{-1}=5 D_{r}^{-1}$. Each is diffusive at large length and time scales.

In what follows we review (Sec. 2) the general physics of motile particles, focusing on two simple models, inspired respectively by bacteria and by synthetic colloidal swimmers. We then address their many body physics in general (Sec. 3), and MIPS in particular, first within a local approximation whereby the swim speed depends on density but not its gradients (Sec. 4). After exploring the aspects of MIPS for which this approximation is sufficient (Secs. 5,6) we move beyond it, showing in Sec. 7 that a careful consideration of nonlocal or gradient terms gives dynamics that is, after all, not equivalent to any form of passive phase separation. We conclude briefly in Sec. 8 .

\section{MOTILE PARTICLES}

\subsection{Run-and-Tumble Bacteria and Active Brownian Particles}

We start by considering two limiting models of the stochastic dynamics of a single active particle (Fig.1). The first is a so-called 'run-and-tumble particle' (RTP), whose motion consists of periods of persisent swimming motion, called 'runs', punctuated by sudden changes of direction, called 'tumbles' $(38,41)$. This is a canonically simplified model of the dynamics of bacteria such as $E$. coli. It supposes the runs to be straight lines, traversed with fixed speed $v$, and punctuated at random by instantaneous tumbles, occurring at some fixed rate $\alpha$, each of which completely decorrelates the swimming direction. At time- and length-scales much larger than $\alpha^{-1}$ and $\ell \sim v / \alpha$, this motion is a diffusive random walk. ${ }^{1}$ It is a simple exercise to calculate its diffusivity in $d$ dimensions as $D=v^{2} / \alpha d$.

Our second model is called an active Brownian particle (ABP) (10). This also has a fixed swim speed $v$, but its direction decorrelates smoothly via rotational diffusion, with angular diffusivity $D_{r}$. This rotation is typically thermal, hence the 'Brownian' label: an instance is self-phoretic colloids, which asymmetrically catalyze conversion of a surrounding fuel to create self propulsion along an axis that slowly rotates by angular Brownian motion. (Another instance is E. coli mutants, called 'smooth swimmers', which have lost the ability to tumble.) At large length- and time-scales the motion is again a diffusive random walk; finding the diffusivity is another easy exercise with the result $D=v^{2} / d(d-1) D_{r}$.

\footnotetext{
${ }^{1}$ As so far described, this model is nothing but the Lorentz gas introduced to model electron transport in metals. Under the assumption that background atoms are random immobile scatterers and that the electron-atom interactions amount to elastic scattering, electrons indeed undergo runand-tumble motion (42).
}

RTP:

Run-and-Tumble Particle

ABP: Active Brownian Particle PBP: Passive Brownian Particle 
As shown in (43), we can generalize these two calculations of the large scale diffusivity $D$ to include a superposition of the ABP and RTP dynamics, and also to include a purely thermal direct contribution $D_{t}$ to the translational diffusivity. The result is

$$
\begin{aligned}
D & =\frac{v^{2} \tau}{d}+D_{t} \\
\tau^{-1} & =\alpha+(d-1) D_{r}
\end{aligned}
$$

where $\tau$ is the orientational relaxation time of the active particle. Note that the $D_{t}$ contribution is often negligible compared to the active part; it is frequently set to zero in simulation studies, and will sometimes be silently omitted in what follows.

\subsection{Spatial Variations in Motility Parameters: a mesoscopic approach}

The above results establish a prima-facie connection between a broad generic class of active particle dynamics (with RTP and ABP as limiting cases) and the physics of isothermal passive Brownian particles (PBPs): after suitable coarse-graining, all describe diffusive random walks of the type canonically exemplified by PBPs. At first sight, the effect of activity is simply to increase the diffusivity $D$ from that of the equivalent passive particle, typically by a large factor. However, a subtler aspect of the connection to PBPs is revealed if one allows $\tau$ and $v$ to be functions of the particle's position, $\mathbf{r}(38,40)$. For instance, a nontrivial $v(\mathbf{r})$ would arise for bacteria swimming in a polymer gel of variable strand density, so propulsion is more effective in some regions than others. An explicit coarse-graining (43) of the microscopic dynamics gives in this case the following equations for the probability density $\varphi(\mathbf{r})$ of our single active particle and its flux $\mathbf{j}$ :

$$
\begin{aligned}
\dot{\varphi} & =-\nabla \cdot \mathbf{j} \\
\mathbf{j} & =-D \nabla \varphi+\mathbf{V} \varphi \\
D(\mathbf{r}) & =\frac{v(\mathbf{r})^{2} \tau(\mathbf{r})}{d} \\
\frac{\mathbf{V}(\mathbf{r})}{D(\mathbf{r})} & =-\nabla \ln v(\mathbf{r})
\end{aligned}
$$

To simplify the form of these equations, we have set $D_{t}=0$. They are the same equations as one would write down to describe a PBP, with a spatially varying $D=v^{2} \tau / d$, except for the presence of an extra drift velocity $\mathbf{V}$. This drift velocity is equivalent to an external potential $\beta U(\mathbf{r})=\ln v(\mathbf{r})$, where $\beta \equiv 1 / k_{B} T$. The active particle behaves as an isothermal $\mathrm{PBP}$ in the presence of this effective potential, which derives solely from activity (38). Its steady-state probability density accordingly obeys a Boltzmann-like distribution

$$
\varphi_{s s} \propto \exp [-\beta U]=\frac{1}{v(\mathbf{r})}
$$

Restoring nonzero $D_{t}$ introduces a factor $\left(1+D_{t} d / v^{2} \tau\right)^{-1}$ in the r.h.s of Eq. 9. So long as $v(\mathbf{r})$ is the only non-constant parameter, this leads to $\varphi_{s s} \propto 1 / \sqrt{v^{2} \tau+d D_{t}}$. For small $D_{t}$ this changes slightly the form of Eq. 10, but not its qualitative physics.

The effective potential $U$ emerges under conditions where there is no actual force field acting on the particle. Hence an active origin for particle diffusion causes deviations from the Boltzmann distribution, $\varphi_{s s} \propto \exp [-\beta H]$, that cannot be absorbed by any global rescaling 


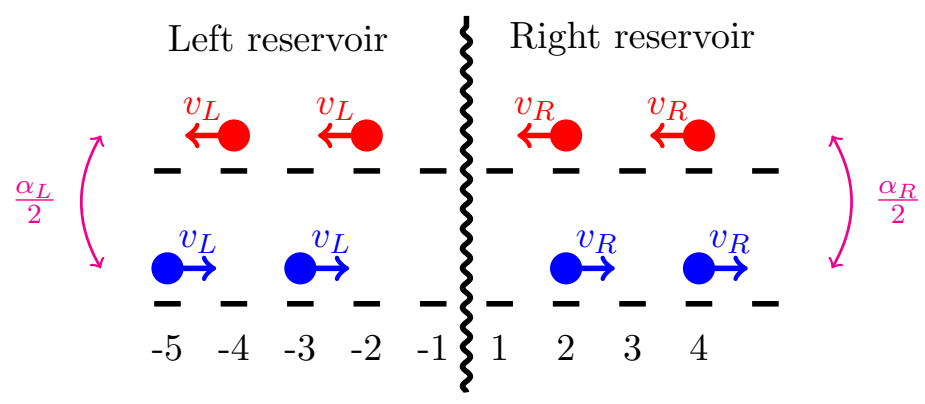

Figure 2

Schematic representation of two reservoirs (left and right) containing ideal lattice-gases of RTPs in $d=1$ with different speeds $v_{L}$ and $v_{R}$. The kinetic flux across the boundary is $v_{L} \rho_{-1}^{+}-v_{R} \rho_{1}^{-}$,

where $\rho_{i}^{ \pm}$is the mean number of particles moving to the right or to the left on site $i$. (These are shown separately in the figure for the sake of clarity.) In steady-state, where densities are uniform and symmetric within each reservoir, the vanishing of the kinetic flux imposes $\rho_{L} v_{L}=\rho_{R} v_{R}$.

of temperature. (Here $H$ is the actual Hamiltonian, not incorporating $U$.) The situation is somewhat like a PBP in a bath at non-uniform temperature, so that its (root-mean-square) speed $\bar{v}$ depends on its position (40). Indeed, if one has a box of passive ideal gas particles, in which two halves of the box are maintained at unequal temperature, then crudely equating the kinetic particle fluxes from one section to the other across the interface requires equality of $\rho \bar{v}$, not of the density $\rho$. In steady state one therefore recovers $\rho \propto 1 / \bar{v}$ which is the direct counterpart of Eq. 10 for a passive, but non-isothermal, system.

We now give a quantitative version of this argument for an active ideal 1D lattice gas (Fig. 2). Here left $(L)$ and right $(R)$ boxes of RTPs, having different swim speeds $\left(v_{L}, v_{R}\right)$ and tumble rates $\left(\alpha_{L}, \alpha_{R}\right)$, are put in contact. (Within each box, left- and right-moving particles have the same speed and tumble rate; the notation differs from (37).) Calling $\rho_{i}^{ \pm}$ the mean number of particles on site $i$ going to the right $(+)$ and to the left $(-)$ we have

$$
\begin{aligned}
\forall i \neq 1 & \dot{\rho}_{i}^{+}=v_{L / R}\left(\rho_{i-1}^{+}-\rho_{i}^{+}\right)+\alpha_{L / R}\left(-\rho_{i}^{+}+\rho_{i}^{-}\right) / 2 \\
\forall i \neq-1 & \dot{\rho}_{i}^{-}=v_{L / R}\left(\rho_{i+1}^{-}-\rho_{i}^{-}\right)+\alpha_{L / R}\left(\rho_{i}^{+}-\rho_{i}^{-}\right) / 2 \\
& \dot{\rho}_{1}^{+}=v_{L} \rho_{-1}^{+}-v_{R} \rho_{1}^{+}+\alpha_{R}\left(-\rho_{1}^{+}+\rho_{1}^{-}\right) / 2 \\
\dot{\rho}_{-1}^{-} & =v_{R} \rho_{1}^{-}-v_{L} \rho_{-1}^{-}+\alpha_{L}\left(\rho_{-1}^{+}-\rho_{-1}^{-}\right) / 2
\end{aligned}
$$

In steady-state, Eq. 11 and Eq. 12 impose a constant density within each bulk:

$$
\forall i \geq 1 \quad \rho_{-i}^{+}=\rho_{-i}^{-} \equiv \rho_{L} ; \quad \rho_{i}^{-}=\rho_{i}^{+} \equiv \rho_{R}
$$

The boundary relations Eq. 13, Eq. 14 then require $v_{L} \rho_{L}=v_{R} \rho_{R}$, in order to balance the kinetic fluxes from the two reservoirs across the interface. This is a simple instance of the more general result $\rho \propto 1 / v$, and also explains qualitatively why spatial variations of $\tau$ have no effect on steady states: $\tau$ enters the expression for the diffusivity, but not directly that for the local flux balance between our two compartments. Note however that for RTPs with finite tumble duration $\Delta$, Eq. 6 and 7 hold up to the rescaling $(v, \alpha) \rightarrow(v, \alpha) /(1+\alpha \Delta)(37)$. The tumbling rate $\alpha$ (and thus $\tau$ ) then enters the steady-state distribution since it controls the kinetic flux through the rescaling of the velocity. This finite- $\Delta$ correction could easily be retained, but is ignored for simplicity from now on. 


\section{MANY-BODY PHYSICS}

\subsection{Dynamics of the Collective Density}

Above we addressed a single particle, whose probability density evolves deterministically via the diffusion-drift equations, Eq. 6-Eq. 9. Since these equations also describe a PBP in an external potential, they are already familiar, and one can use standard and well-tested procedures (44) to derive from them a stochastic equation of motion for the coarse-grained density $\rho(\mathbf{r})$ in a system of many particles. Note that this $\rho$ is not a probability density (which would evolve deterministically) but a coarse grained version of the microscopic density operator $\sum_{i=1}^{N} \delta\left(\mathbf{r}-\mathbf{r}_{i}\right)$, which obeys a stochastic equation of motion. We state the result first for a collection of noninteracting active particles in an environment of spatially varying motility parameters, $v(\mathbf{r}), \tau(\mathbf{r})(37)$ :

$$
\begin{aligned}
& \dot{\rho}=-\nabla . \mathbf{J} \\
& \mathbf{J}=-D \nabla \rho+\mathbf{V} \rho+\sqrt{2 D \rho} \boldsymbol{\Lambda}
\end{aligned}
$$

Here $D(\mathbf{r})$ and $\mathbf{V}(\mathbf{r})$ obey Eqs. 8, 9, and $\boldsymbol{\Lambda}$ is a vector-valued unit white noise. The multiplicative noise term is to be read in the Ito sense, which means that Eq. 17 is viewed as the small-timestep limit of a discrete process in which the noise term is evaluated at the start of each timestep (45). Another possible choice would be the Stratonovich convention which requires the noise to be evaluated mid-way during the timestep. However, switching to this convention introduces an additional drift velocity into Eq. 9 which is present even for passive particles (45), making it harder to identify the specific effects of activity. It also makes it harder to generalize to the interacting case, which is our next task.

\subsection{Density-Dependent Motility Parameters}

Once the Itō choice is made, Eq. 16 and 17 can painlessly be generalized to the case where the dependence of diffusivity $D$ and drift velocity $\mathbf{V}$ on spatial position $\mathbf{r}$ is in fact caused by a dependence of the motility parameters $v$ and $\tau$ on the density of particles in that neighborhood (37). Of course, this is not the only type of interaction possible: for instance, hard-core collisions between ABPs are not directly of this form, but can be partly approximated by it as we shall see in Sec. 5.2 below. On the other hand, bacteria can respond to their local density via a biochemical pathway (quorum sensing (31)), and in some cases this response is linked directly to their motility $(46,47)$. Hence it is natural to address the case where the motility parameters directly depend on the coarse-grained density $\rho$. Given this choice of interaction, we can write

$$
\begin{aligned}
D([\rho], \mathbf{r}) & =\frac{v([\rho], \mathbf{r})^{2} \tau([\rho], \mathbf{r})}{d} \\
\frac{\mathbf{V}([\rho], \mathbf{r})}{D([\rho], \mathbf{r})} & =-\nabla \ln v([\rho], \mathbf{r})
\end{aligned}
$$

where the new argument $[\rho]$ denotes an arbitrary functional dependence on the coarsegrained density field $\rho(\mathbf{r})$, and we have again set $D_{t}=0$, thereby neglecting the direct Brownian contribution to translational diffusivity. Eq. 16 and Eq. 17 still apply, except that an additional term $\rho\left(\nabla \frac{\delta}{\delta \rho(\mathbf{r})} D([\rho], \mathbf{r})\right)$ appears on the right side of Eq. 17. In fact this term vanishes in most cases of interest, one exception being the asymmetric lattice model described in Sec. 5.1 below (37), and we will not discuss it further.

8 Michael Cates and Julien Tailleur 
To see whether this description of interacting motile particles is still equivalent to a set of PBPs in thermal equilibrium, we next consider the Fokker-Planck equation for the many-body probability $P[\rho]$, which reads:

$$
\dot{P}[\rho]=-\int d \mathbf{r}\left(\nabla \frac{\delta}{\delta \rho(\mathbf{r})}\right)\left[\mathbf{V} \rho-D \nabla \rho-D \rho\left(\nabla \frac{\delta}{\delta \rho(\mathbf{r})}\right)\right] P[\rho]
$$

We may now define an 'equilibrium-like' steady state $P_{\text {eq }}[\rho]$ as one in which the probability current vanishes:

$$
\mathcal{J}\left[P_{\mathrm{eq}}\right]=\left[\mathbf{V} \rho-D \nabla \rho-D \rho\left(\nabla \frac{\delta}{\delta \rho(\mathbf{r})}\right)\right] P\left[\rho_{\mathrm{eq}}\right]=0
$$

Using the ansatz $P_{\text {eq }}=\exp [-\beta \mathcal{F}]$, one sees by inspection of Eq. 21 that such a flux-free solution exists so long as the following integrability condition is obeyed:

$$
\frac{\mathbf{V}([\rho], \mathbf{r})}{D([\rho], \mathbf{r})}=-\beta \nabla \frac{\delta \mathcal{F}_{\mathrm{ex}}}{\delta \rho}
$$

which can be rewritten as

$$
k_{B} T \ln v([\rho], \mathbf{r}) \equiv \mu_{\mathrm{ex}}([\rho], \mathbf{r})=\frac{\delta \mathcal{F}_{\mathrm{ex}}}{\delta \rho}
$$

This condition requires that the functional defined here as $\mu_{\mathrm{ex}}([\rho], \mathbf{r})$ is itself the derivative of some other functional $\mathcal{F}_{\text {ex }}$. There is no general reason for this to hold.

Nonetheless, if it does hold, our system of interacting active particles is dynamically equivalent, at large length and time scales, to a PBP fluid with the free energy functional:

$$
\mathcal{F}[\rho]=k_{B} T \int \rho(\ln \rho-1) d \mathbf{r}+\mathcal{F}_{\text {ex }}[\rho]
$$

Here the integral can be viewed as an ideal entropy contribution and the excess part would, for real PBPs, be caused by some interaction Hamiltonian. For our active particles, it stems instead from the density-dependent swim speed $v([\rho], \mathbf{r})$. Just as in the one-body problem, any density-dependence of the angular relaxation time $\tau([\rho], \mathbf{r})$ plays no role in $\mathcal{F}$.

Note that the zero-flux condition, Eq. 21, which decides the existence of a steady-state mapping onto a thermal equilibrium system, cannot be derived without the proper noise terms in Eq. 17. (These set the prefactor of the second functional derivative in Eq. 20 to be $D \rho$ and hence lead to the condition Eq. 23.) Unless drift and noise terms are handled on equal terms, for instance when addressing higher order gradient terms of the type discussed in Sec. 7, one cannot be sure whether such a mapping still exists or not (48).

When it exists, $\mathcal{F}[\rho]$ specifies not just the most probable configuration of $\rho(\mathbf{r})$ but also its entire spectrum of steady-state fluctuations, thus taking us far beyond the linear stability analysis sketched in the introduction. But, as stated already, one cannot generally expect Eq. 23 to hold true. When it doesn't, one can still formally define some functional $\mathcal{F}=-k_{B} T \ln P_{\text {stat }}[\rho]$, but this no longer governs a flux-free solution of Eq. 20 and is thus not equivalent to any choice of equilibrium dynamics. This restates the fact that coarse-graining cannot create a general equivalence between active systems, which rely on microscopically irreversible dynamics, and passive systems, which obey detailed balance at all scales. Suppose however that $\rho$ is slowly varying in space and that the swim speed 
$v([\rho], \mathbf{r})$ depends isotropically on the values of $\rho$ within some finite range of the point $\mathbf{r}$. Under these conditions a Taylor expansion gives (32)

$$
v([\rho], \mathbf{r})=v(\rho(\mathbf{r}))+\mathcal{O}\left(\nabla^{2} \rho\right)
$$

Suppressing the gradient contribution is equivalent to assuming a perfectly local functional dependence of swim speed on density: $v=v(\rho)$. As detailed below, it is easy to confirm that $\mathcal{F}_{\text {ex }}$, and hence the mapping onto equilibrium, does exist in this case (37). This is a limiting approximation, whose physicality is not guaranteed. However, much can be gained by assuming the local approximation, finding the consequences, and then returning to address gradient effects. The literature has followed this path and we do so here. ${ }^{2}$

\section{THE LOCAL APPROXIMATION}

\subsection{Without passive Brownian diffusion $D_{t}=0$}

In the local approximation, $v(\mathbf{r})=v(\rho(\mathbf{r}))$ as just described. The nonequilibrium excess chemical potential $\mu_{\mathrm{ex}}=k_{B} T \ln v$ then always obeys the integrability condition, Eq. 23 with $\mathcal{F}_{\text {ex }}(\rho)=\int_{0}^{\rho} \ln v(s) d s$. The system is equivalent to interacting PBPs with

$$
\begin{aligned}
\mathcal{F}[\rho] & =\int f(\rho) d \mathbf{r} \\
\beta f(\rho) & =\rho(\ln \rho-1)+\int_{0}^{\rho} \ln v(s) d s
\end{aligned}
$$

Since all steady state statistics depend only on the product $\beta \mathcal{F}[\rho]$, it should by now be clear that we can set $\beta=1$ without loss of generality, and we do this silently from now on. The chemical potential may then be written as a sum of ideal and excess parts

$$
\mu=\frac{\delta \mathcal{F}}{\delta \rho(\mathbf{r})}=\mu_{\mathrm{id}}+\mu_{\mathrm{ex}}=\ln \rho(\mathbf{r})+\ln v(\rho(\mathbf{r}))
$$

It should be noted that, within the premise of interactions whose sole effect is to make $v$ and $\tau$ depend on density, Eqs. 26-28 involve no approximation beyond the locality of $v(\rho)$ and the validity of the coarse-graining leading to the mesoscopic equations 16-20.

Nonetheless, we first proceed within the context of a (Landau-like) mean-field theory in which spatial fluctuations are ignored, performing a global minimization of the function $F=\sum_{i} V_{i} f\left(\rho_{i}\right)$ at constant volume $\sum_{i} V_{i}$ and constant particle number $\sum_{i} V_{i} \rho_{i}$ : phase separation then arises whenever more than one $V_{i}$ is nonzero. An important caveat is that this makes sense only if the interfacial tension between phases is finite, since this tension alone prevents the macroscopic phases from fragmenting into uncountably many small domains at the (nominally) coexisting densities. This means that the global minimization, while not making direct use of gradient terms, tacitly assumes that these terms exist and do not violate Eq. 23. We proceed on that basis, but revisit this issue in Sec. 7.

For two-phase coexistence, the global minimization proceeds just as in equilibrium: one first searches for concavities in the function $f(\rho)$, and where these exist performs a

\footnotetext{
${ }^{2}$ Should this route not be to the reader's taste, she may skip forward to Sec. 7.1 and then return. That section offers the simplest (phenomenological) treatment of nonlocality, within which all the main results of Sec. 4 still apply. 

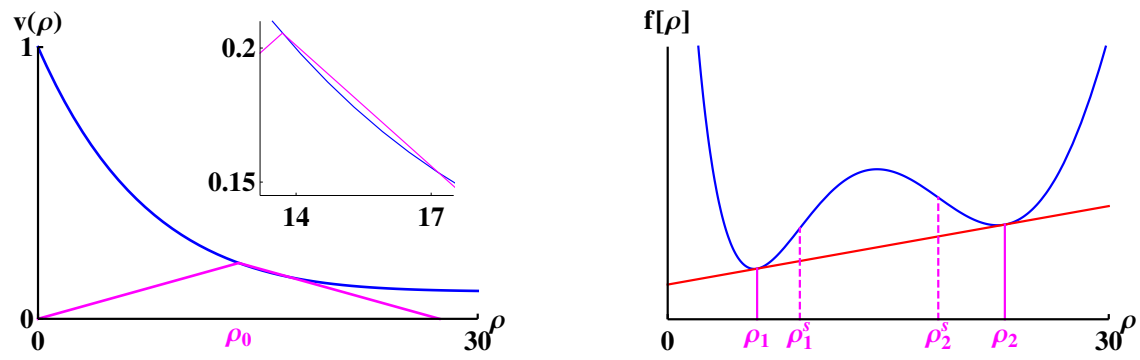

Figure 3

Construction of the effective free energy density $f(\rho)$ in the mapping from active particles with strictly local motility interactions onto a fluid of interacting Brownian particles. If $v(\rho)$ decreases rapidly enough (left) the resulting $f(\rho)$ has a negative curvature (spinodal) region (right) with the global equilibrium state comprising a coexistence of the binodal densities $\rho_{1}, \rho_{2}$. The condition for instability $\left(f^{\prime \prime}<0\right)$ translates into the geometric construction shown on $v(\rho)$ : draw a line from the origin to any point on the curve and reflect this line in the vertical axis. If the slope of $v(\rho)$ is less than the reflected line, the system is unstable. The figures correspond to $v(\rho)$ given by Eq. 32 with $D_{t} \simeq 1.410^{-3}, v_{0}=1, v_{1}=0.1, \varphi=4, \tau=2, d=2$ and $2 \rho_{0}=\rho_{1}^{s}+\rho_{2}^{s}$.

common-tangent construction. This construction finds unique coexisting densities $\rho_{1}$ and $\rho_{2}$, such that the chemical potential $\mu=d f / d \rho$ in the two phases are equal, and such that their thermodynamic pressures $p=\mu \rho-f$ are also equal. The first of these equalities requires equal slopes for the tangents to $f(\rho)$ at the coexisting densities, whereas the second requires equal intercepts: thus a single tangent connects both coexistence densities (Fig. 3 ). The condition for concavity, $f^{\prime \prime}(\rho)<0$ (where primes now denote $\rho$ derivatives), implies $v^{\prime}(\rho)<-v / \rho$, which is exactly the condition for linear instability found in Eq. 3. The set of such negative-curvature points defines the spinodal region, a familiar concept in mean-field thermodynamics (49). The common-tangent construction also encloses zones where $f$ has positive curvature; here phase separation occurs by nucleation and growth (50).

This approach thus shows how self-propelled particles with no attractions but a decreasing $v(\rho)$ can be mapped, at a (Landau-like) mean-field level of global minimization, to a system of attractive PBPs undergoing equilibrium liquid-gas phase separation.

\subsection{The effect of finite thermal diffusivity: $D_{t} \neq 0$}

We have just outlined the theory of motility-induced phase separation (MIPS) as first presented in (37) for RTPs, and extended later to include continuous angular diffusion in (43), thereby embracing ABPs also. For simplicity we neglected the Brownian translational contribution $D_{t}$; restoring this (as discussed after Eq. 10 above) gives the modified integrability condition

$$
\frac{\tau v \nabla v}{v^{2} \tau+d D_{t}}=\nabla \frac{\delta}{\delta \rho} \mathcal{F}_{\mathrm{ex}}
$$

For a strictly local $v(\rho(\mathbf{r}))$, a sufficient condition for $\mathcal{F}_{\text {ex }}$ still to exist is that only the speed (not $\tau$ or $D_{t}$ ) depends on the density. One then find an excess free energy

$$
\mathcal{F}_{\text {ex }}=\int f_{\text {ex }}(\rho(\mathbf{r})) d \mathbf{r} \quad \text { with } \quad f_{\text {ex }}(\rho)=\int^{\rho} \frac{1}{2} \ln \left[v^{2}(s) \tau+d D_{t}\right] d s
$$



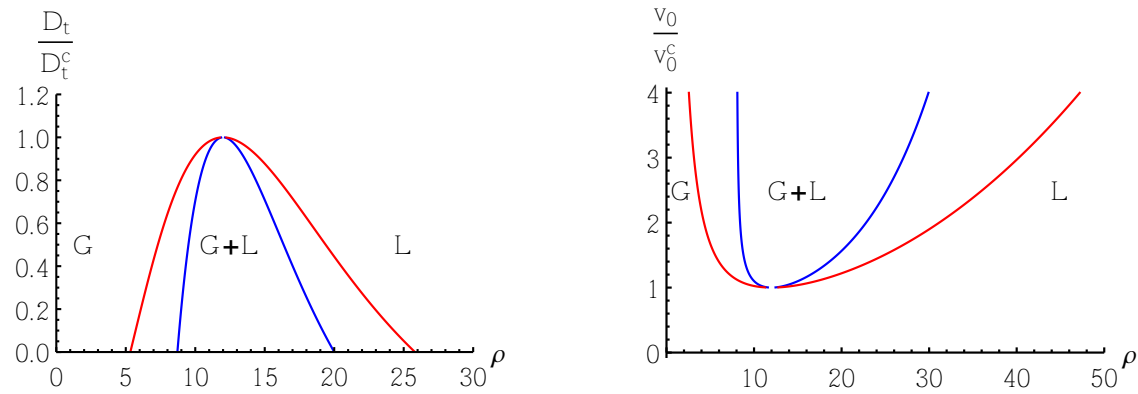

Figure 4

Liquid-gas phase diagrams of MIPS for $v(\rho)$ as defined in Eq. 32 with $v_{0}=1, v_{1}=0.1, \varphi=4$, $d=2, \tau=1$ (left) and $D_{t}=0.25, v_{1}=0.25, \varphi=4, d=2, \tau=1$ (right). Red and blue lines correspond to binodal and spinodal curves.

Again, the non-convexity of the free energy density $f(\rho)=\rho(\ln \rho-1)+f_{\text {ex }}(\rho)$ signals the possibility of MIPS; the spinodal region corresponds to

$$
f^{\prime \prime}(\rho)<0 \quad \Leftrightarrow \quad v^{2} \tau\left(1+\rho \frac{v^{\prime}}{v}\right)<-d D_{t}
$$

For $D_{t}=0$, one recovers Eq. 3, whereas a finite $D_{t}$ makes the system more stable by tending to smooth out density fluctuations. In particular, when $v \rightarrow 0$, the modified condition Eq. 31 is never fulfilled if $D_{t}$ is finite. For any given $v(\rho)$, there is thus a minimal ratio $v^{2} \tau / d D_{t}$ of active to thermal diffusivities below which MIPS never occurs.

As an example, let us consider a propulsion speed decaying exponentially from a value $v_{0}$ to a smaller one $v_{1}$, on some characteristic density scale $\varphi$ :

$$
v(\rho)^{2}=v_{0}^{2}+\left(v_{1}^{2}-v_{0}^{2}\right)\left(1-e^{-\rho / \varphi}\right)
$$

With this choice, $f_{\mathrm{ex}}(\rho)=\rho \ln \left[v_{1}^{2} \tau+d D_{t}\right] / 2-\varphi\left[\operatorname{Li}_{2}(-A)-\mathrm{Li}_{2}\left(-A e^{-\rho / \varphi}\right)\right] / 2$ where $\operatorname{Li}_{2}(z)$ is the polylogarithm function of order 2 and $A=\left(v_{0}^{2} \tau-v_{1}^{2} \tau\right) /\left(v_{1}^{2} \tau+d D_{t}\right)$. The term in $f_{\text {ex }}$ that is linear in $\rho$ plays no role in stability or phase equilibria. The spinodals $\left(f^{\prime \prime}(\rho)=0\right)$ are roots of $\lambda \exp \lambda=-2 e^{2} / A$, where $\lambda \equiv 2-\rho / \varphi$, which exist only when $A \geq 2 e^{3} / 3$. For a given $v(\rho)$, this sets a maximal value for $D_{t}$

$$
D_{t}^{c}=\frac{v_{0}^{2} \tau-v_{1}^{2} \tau\left(1+2 e^{3}\right)}{2 d e^{3}}
$$

at which MIPS ends, presumably at a critical point. Conversely, for given $v_{1}$ and $D_{t}$, there is a minimum bare swim speed $v_{0}^{c}$ for MIPS, obeying

$$
v_{0}^{c}=\sqrt{v_{1}^{2}\left(1+2 e^{3}\right)+2 e^{3} \frac{D_{t} d}{\tau}}
$$

The spinodal and coexistence lines for $v(\rho)$ obeying Eq. 32 are shown in Fig. 4, while Fig. 5 gives those for an even simpler form of $v(\rho)$, which features below (see Eq. 39):

$$
v(\rho)=v_{0}\left(1-\rho / \rho^{*}\right)
$$



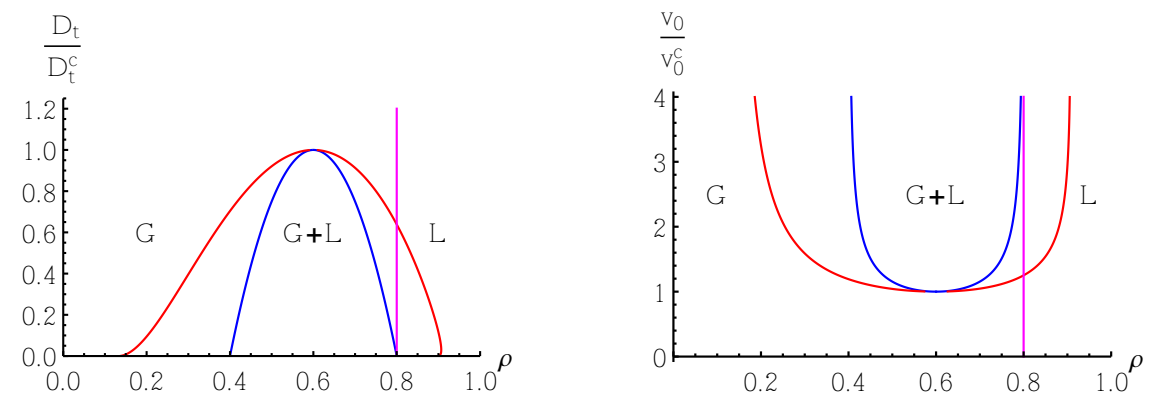

Figure 5

Phase diagrams of MIPS for $v(\rho)$ as defined in Eq. 35 with $v_{0}=5$ (left), $D_{t}=0.5$ (right), $d=2$, $\tau=1$ and $\rho^{*}=0.8$ (vertical line). Red and blue lines are binodal and spinodal curves.

Again the excess free energy can be computed exactly ${ }^{3}$ as can the spinodals:

$$
\rho_{s}^{ \pm}=\rho^{*}(3 \pm \sqrt{1-8 \epsilon}) / 4
$$

where $\epsilon=d D_{t} / v_{0}^{2} \tau$ is a ratio of passive and active diffusivities. MIPS thus exists only when $\epsilon<1 / 8$, i.e.

$$
D_{t}<D_{t}^{c} \equiv \frac{v_{0}^{2} \tau}{8 d} ; \quad \text { or } \quad v_{0} \geq v_{0}^{c} \equiv \sqrt{\frac{8 d D_{t}}{\tau}}
$$

Note that Eq. 35 for $v(\rho)$, and also the resulting phase diagrams, are only meaningful for $\rho \leq \rho^{*}$, beyond which the system reverts to a passive state (vertical lines in Fig. 5).

The study of the spinodals and the limit of existence of MIPS below a critical velocity can also be derived through a linear stability analysis of continuum mean-field equations $(35,51)$. Predicting the binodals, however, requires the derivation of the effective free energy.

\section{NUMERICAL EVIDENCE FOR MIPS}

\subsection{Phase Separation in Run-and-Tumble Particles}

Clear numerical evidence for MIPS within the region where $v^{\prime}<-v / \rho$ (Eq. 3) was presented in (37) for simulations of RTPs in $d=1$ dimension. In these simulations, a fixed coarsegraining length is used to define the density $\rho$ upon which $v$ then depends locally. The coexisting densities were compatible, within numerical error, with those set by the commontangent construction.

It is known that for the equivalent system of PBPs with attractions, phase separation cannot proceed to completion in $d=1$, because domain walls have a finite energy cost $E$. The equilibrium state thus has a domain-wall density $\propto \exp [-\beta E]$. While this precludes long-range order, for large $E$ the 'phase-separated' state (containing sparse domain walls between patches of $\rho_{1}$ and $\rho_{2}$ ) is unambiguously different in appearance from the 'singlephase' regime in which there are only small density fluctuations about an average value. The 1D simulations of (37) observed phase separation in this restricted sense; domain walls were formed and slowly became more dilute through a coalescence process.

\footnotetext{
${ }^{3}$ Specifically, $f_{\mathrm{ex}}=\frac{\rho}{2}\left(\ln \left(v_{0}^{2} \tau\right)-2\right)+\frac{\rho-\rho^{*}}{2} \ln \left[\epsilon+\left(1-\frac{\rho}{\rho^{*}}\right)^{2}\right]-\rho^{*} \sqrt{\epsilon} \arctan \left(\frac{1-\frac{\rho}{\rho^{*}}}{\sqrt{\epsilon}}\right)$.
} 
Microscopic simulations of RTPs with crowding interactions were subsequently presented in $d=1$ and $d=2$ using a fast lattice-based discretization (32). In $d=1$ the hopping rate of right-going $(+)$ and left-going $(-)$ particles on site $i$ can be chosen as

$$
v^{ \pm}(i,[\rho])=v_{0}\left(1-\frac{\tilde{\rho}_{i}}{\rho_{M}}\right) ; \quad \text { with } \quad \tilde{\rho}_{i}=\sum_{j} K_{i j}^{ \pm} \rho_{j}
$$

Here $\rho_{j}$ is the number of particles on site $j, \rho_{M}$ controls the maximal number of particles on each site, and $K_{i j}$ is a kernel describing how particles on site $i$ interact with those on site $j$. When $K_{i j}$ is a smooth symmetric kernel, particles are equally sensitive to the particles in front of them or behind them. (This seems appropriate for chemically mediated interactions.) Conversely, for asymmetric kernels the particles are more sensitive to those in front of them; the limiting case $K_{i j}^{ \pm}=\delta_{i \pm 1, j}$ corresponds to a partial exclusion process, which is a good lattice-based model of steric crowding $(52,53)$.

A fluctuating hydrodynamic description akin to Eqs. 16-17 can be derived to account for the large scale behavior of this system (32). This shows a mapping to an equilibrium system of passive particles with attractive interactions. Indeed the system admits a free energy functional $\mathcal{F}$ which predicts MIPS exactly as presented in Sec. 4 (32). Homogeneous profiles are predicted to be linearly unstable only at rather high densities $\left(\rho>\rho_{M} / 2\right)$ but nucleation can occur at a much lower ones (32). In $d=1$, MIPS again creates alternating sequences of high- and low-density domains, separated by domain walls. (The limiting case $\rho_{M}=1$ with $K_{i j}^{ \pm}=\delta_{i \pm 1, j}$ has been studied in detail in (54).)

Eq. 38 can easily be generalized to higher dimensions and we now turn to the $2 \mathrm{D}$ case. For symmetric kernels $K_{i j}$, in the limit of large $\rho_{M}$, the mean-field free energy analysis predicts quantitatively both the occurrence of complete phase-separation and the values of the coexisting densities (32). When $\rho_{M}$ is finite, MIPS still occurs but (as is common for partial exclusion models (53)) the coexisting densities are not those predicted by mean-field theory. The case of an asymmetric kernel, which might be thought to mimic steric exclusion on a lattice more closely, has a richer phenomenology. MIPS occurs, but the coexisting densities are incorrectly predicted by the common-tangent construction even when meanfield theory might be expected to hold $\left(\rho_{M} \rightarrow \infty\right)$. For instance, the gas density in the phase coexistence region, given by the lower binodal, goes to zero as $v_{0} \rightarrow \infty$ (32) while the theory of Sec. 4 predicts a non-vanishing saturation vapor density in this limit.

Furthermore, for the asymmetric kernel $K_{i j}^{ \pm}=\delta_{i \pm 1, j}$, MIPS is only seen for large enough values of $\ell=v_{0} \tau$; when this is less than about four times the repulsion radius (four lattice sites), homogeneous profiles are instead stable. This is related to, but distinct from, the minimum-speed requirement for off-lattice MIPS found above in Sec. 4. In the lattice models, the coarse-grained theory of MIPS fails to capture the minimal run-length, not because $D_{t}$ is neglected, but because of the short-scale breakdown of the diffusive approximation itself (Eq. 16 and 17). By formulating a mean-field theory directly at the microscopic lattice level (32), a minimum speed criterion is recovered. This breakdown of MIPS was seen for general anisotropic kernels $K$, but not for rotationally symmetric ones. Since these kernels differ only at the level of gradient terms so far neglected, this points to a more important role for such terms than in equilibrium problems (see Section 7 ).

In all 2D systems where MIPS is seen, an initially homogeneous profile in the spinodal region gives way to a droplet domain morphology whose length scale $L(t)$ coarsens with a power law in time: $L(t) \sim t^{1 / 3}$, see Fig.6. This is the classically expected result for diffusive coarsening, without coupling to a momentum-conserving fluid, in a passively phase- 

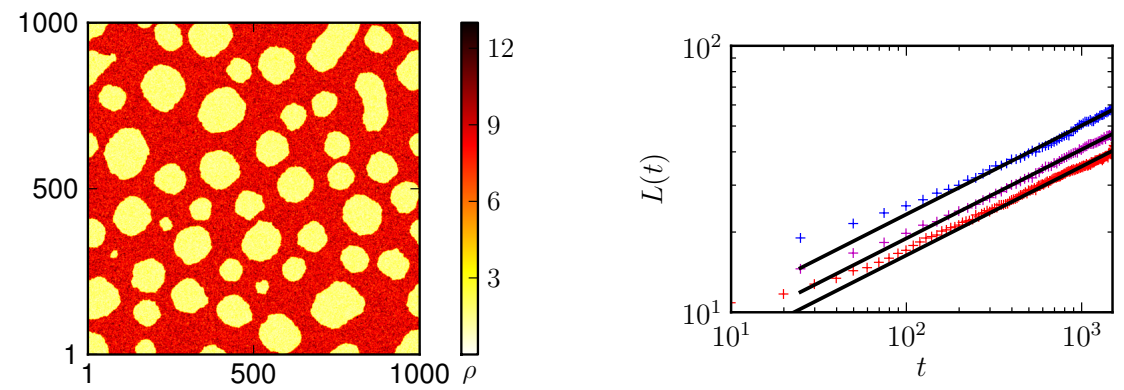

Figure 6

(Left) A 2D run-and-tumble system undergoing MIPS. Simulated via a lattice model $(1000 \times 1000$ sites) as detailed in (32), with local density (particles per site) color-coded on scale at right.

(Right) Log-log plot of domain scale $L(t)$ for droplet coarsening within the spinodal region. Solid lines have slope $1 / 3$. Results for three different kernels relating $v$ to the density are shown for $200 \times 200$ site lattices (adapted from (32), courtesy of A. Thompson).

separating system (50). The exponent can be explained by considering the diffusive flux down chemical potential gradients set by the Laplace pressure differences across curved interfaces of radius $L$ and fixed interfacial tension. Since this tension vanishes within the local approximation, we defer further discussion to Section 7 .

\subsection{Phase Separation in Active Brownian Particles}

Convincing evidence for MIPS is also seen in numerical studies on ABPs for a variety of hard-sphere potentials $(34,35,55,56,57)$, as well as for soft spheres $(33,51)$. Apart from the difference in rotational diffusion dynamics, which is inessential (43), these simulations crucially differ from those on RTPs in which a density-dependent swim-speed is directly encoded into the dynamics. Simulations on ABPs generally instead address hardcore swimming particles, whose $v([\rho], \mathbf{r})$ is not encoded a priori into the equations of motion (an exception being (36)). Instead, collisions can be expected to slow down the particles at high density. One can then define an emergent $v$ as the average of the true particle velocity projected along the propulsion direction. Monitoring this within bulk systems of uniform density $\rho$, one finds $v(\rho)$ decreases almost linearly with density $(33,34,55,56)$ :

$$
v(\rho)=v_{0}\left[1-\rho / \rho^{*}\right]
$$

where $v_{0}$ is the dilute swim speed and $\rho^{*}$ the extrapolated point at which $v$ vanishes altogether. The latter is barely distinguishable from the close packing threshold in both $d=2$ and $d=3(33,55,56,57)$, although this threshold is influenced by activity $(58,59)$, and also by slight softness of the particles in the simulations. Eq. 39 is not only confirmed to high accuracy in simulations but also predicted by various types of kinetic theory $(33,35,51,55)$. Indeed its linear form is easily deduced from a binary collision picture in which a particle stalls for a fixed time interval during each collision.

In contrast to the observed linear behavior of $v(\rho)$, most simulations of spherical ABPs allow no dependence whatsoever of $\tau$, the rotational relaxation time, on density $\rho$. This is because collisions, caused by pairwise central forces between particles, cannot exert torques 
and so cannot rotate the swimming direction. Hence $\tau^{-1}=(d-1) D_{r}$ at all densities. The ABP simulations then confirm that the particle diffusion constant obeys $D(\rho)=v(\rho)^{2} \tau / d+$ $D_{t}$ as expected, with $v(\rho)$ obeying Eq. 39 (33, 34, 35, 55, 56).

To test the relevance of the MIPS theory of Sec. 4.1 for an ABP system, one must note that the density cannot exceed $\rho^{*}$, the close packed value. This stems from hard sphere compressibility constraints that are not encoded in $v(\rho)$ : at high density $v$ is almost zero, and $\mathcal{F}$ for Eq. 27 reverts to that of a highly compressible, passive ideal gas. Thus the mean-field free energy density $f(\rho)=\rho(\ln \rho-1)+\int_{0}^{\rho} \ln v(s) d s$ has to be supplemented by an additional constraint that imposes $\rho \leq \rho^{*}$ (55). Once this is done, both steady-states and coarsening dynamics seen in ABP simulations are in qualitative agreement with the theory of MIPS $(55,56)$. However, some important physics is missing, in that the phase equilibrium predicted from Eq. 39 is independent of the Péclet number, here defined as

$$
\mathrm{Pe}=\frac{3 v_{0} \tau}{\sigma}
$$

where $\sigma$ is the particle diameter. No MIPS is seen numerically for $\mathrm{Pe}<\mathrm{Pe}_{c}$, with $\mathrm{Pe}_{c} \simeq 55$ in $d=2$ and $\simeq 125$ in $d=3$; instead the predicted two-phase region closes off at $\mathrm{Pe}=\mathrm{Pe}_{c}$, probably ending in a critical point $(33,34,55,56,60)$. On the contrary, the MIPS theory of Sec. 4.1 always predicts a spinodal decomposition for $\rho>\rho^{*} / 2$.

Since $\tau$ arises by thermal diffusion (in ABPs), the Péclet number not only governs the ratio of the persistence length $\ell=v_{0} \tau$ of a dilute swimmer to its diameter $\sigma$, but also is of order $v_{0} / v_{0}^{c}$ where $v_{0}^{c}$ is the speed threshold for MIPS set by translational diffusion (see Eq. 37). Thus in experimental ABPs, Pe controls two distinct mechanisms for loss of MIPS: translational thermal diffusion (described in Sec. 4.2) and a small persistence-length to diameter ratio (discussed, for lattice models, in Sec. 5.1). In simulations, however, these can be distinguished by artificially setting $D_{t}=0$. The theory of MIPS laid out in Sec. 4.1 ignores both those effects; it is thus not suprising that this limiting theory thus does not capture the observed disappearance of MIPS in ABPs as Pe is reduced.

The existence of a critical Péclet number $\mathrm{Pe}_{c}$ is consistent with various approaches based on the kinetic theory of gases, suitably adapted to $\operatorname{ABPs}(6,33,34,35,48,51,55,59,60)$. These approaches yield continuum equations whose linear stability analysis can be used to locate spinodals $(33,35,51)$. The results resemble the phase diagrams predicted by the theory of MIPS presented in Sec. 4.2, which allowed for thermal translational diffusion $\left(D_{t} \neq 0\right)$. However, whether a finite $D_{t}$ is indeed what suppresses phase separation at small Pe is questionable. Since Pe $\sim v / v_{0}^{c}$, MIPS should then persist down to Pe of order unity (see Fig.5), thereby under-predicting the reported $\mathrm{Pe}_{c}$ for MIPS by a factor of 50 or more. This is strong evidence that nonzero $D_{t}$ is not solely responsible for the loss of MIPS at low Pe in ABPs. Reinforcing this is the observation that simulations in which $D_{t}$ is set to zero $(33,51)$ give very similar results to those in which $D_{t}$ takes the thermal value set by matching the Brownian mechanism for angular rotation $(34,55,56)$.

One interesting alternative avenue is to use the rate of arrival and departure of ABPs at the surface of a dense domain to compute kinetically the vapor density $\rho_{v}(34,60)$. This approach predicts the existence of a critical Pe. The arrival rate of particles (per unit area) is of order $\rho_{v} v_{0}$ while the departure rate is $\kappa D_{r} / \sigma$, which involves $D_{r}$ because arriving particles must rotate through a finite angle before they can leave. Here $\kappa$ is a dimensionless (fitting) factor that allows for the fact that particles tend to leave in bursts rather than individually. Equating rates gives an expression for the fraction $f_{c}(\rho, \mathrm{Pe})$ of particles in clusters; contours of this function vary as $\mathrm{Pe}^{-1}$, and the limit $f_{c}=0^{+}$closely 
tracks the simulated binodal up to $\mathrm{Pe} \simeq 100$. However, this means $f_{c}(\rho, \mathrm{Pe}) \rightarrow 1$ for all $\rho$ as $\mathrm{Pe} \rightarrow \infty$, so there is no connection in that limit with the phase-separation theory of MIPS that assumes a smooth $v(\rho)$. A partial reconciliation between the two approaches could be to follow (51) and make $\rho^{*}$ in Eq. 39 an explicit function of Pe.

In summary, the 'thermodynamic' approach to MIPS in ABPs (based on assuming a smooth $v(\rho))$ can be qualitatively improved by allowing for finite rotational Péclet number, as in Sec. 4.2. It then yields spinodals in agreement with kinetic theory arguments $(35,51)$. This is however not the main correction needed to account for ABP simulations, which instead stems from the discreteness of the collisional dynamics, as for the lattice model of Sec. 5.1. This can be partially understood using a kinetic approach to cluster growth $(34,60)$ but, so far, this has not been married to the thermodynamic picture, and we do not yet have a complete theory for the critical Péclet number $\mathrm{Pe}_{c}$, below which MIPS disappears.

The region close to the critical point, and any universal exponents associated with it, also remains to be explored (theoretically or numerically), in both $d=2$ and $d=3$. Continuum theories (discussed in Section 7.2 below) could be useful here, as the direct simulations of active particles already require extremely large systems (up to 40 million particles in (56)) to resolve even the non-critical aspects of MIPS that prevail at high Péclet number.

Such aspects include the coarsening law for the domain size $L(t)$ after a quench into the phase-separated regime. In two dimensions this shows an exponent $L(t) \sim t^{\alpha}$ with $0.25 \leq \alpha \leq 0.28(34,55,56,60)$. This is somewhat below the value of $1 / 3$ expected for passive coarsening (and which was also reported in (32) for RTPs in 2D and in (56) for $\mathrm{ABPs}$ in $3 \mathrm{D})$. However it is not yet clear that the difference is numerically significant; it may instead reflect a slow transient approach to an asymptotic $1 / 3$ power (61) (see Section 7.2 below). All in all, the numerical results on $\operatorname{ABPs}(34,55,56,60)$ and RTPs $(32)$ for the coarsening exponents call for complementary studies on larger system sizes. Furthermore, even if the coarsening behavior of ABPs broadly resembles that of a passive system, this is not true when one looks in more detail. For instance in $d=2(55)$ and also in $d=3$ (57), one sees 'lava-lamp' type dynamics in which even fast local fluctuations within a wellseparated domain manifestly break time reversal symmetry. This contrast with the passive case where irreversible dynamics is visible only at scales above $L(t)$.

\section{EXPERIMENTAL IMPLICATIONS}

\subsection{Experiments on Bacteria}

In microbiological studies, formation of dense clusters from a uniform initial population of motile bacterial cells is often encountered (and usually called 'aggregation' rather than phase separation). So far though, a quantitative link between this behavior and MIPS has not been established.

The down-regulation of swimming activity at high density is fundamental to the formation of biofilms (15). A biofilm comprises a region with a high local density of bacteria that are immobilized on a wall or similar support. Biofilms are a widespread problem in health and technology, arising for instance in bacterial fouling of water pipes (13), and lethal infections in patients with cardiac valve implants (14). Biofilm formation generally involves chemical communication between individual cells, but the effect of this may still be representable in part as a density-dependent swim speed $v(\rho)$. Alternatively it is possible to connect the quorum sensing apparatus of bacteria to their self-propulsion mechanism, thereby creating a decreasing $v(\rho)$ directly by genetic engineering $(46,47)$. MIPS might 
also occur by various non-biochemical means, such as simple crowding, hydrodynamically mediated surface accumulation (62), or by secretion of viscosity-enhancing polymers (63).

The MIPS scenario also impacts on the study of pattern formation in bacterial colonies started on an agar plate from a localized source. These were observed before the discovery of quorum sensing (which is a local response to the concentration of a secreted chemical (31)) and attributed instead to long-range chemotactic interactions, which modulate the persistence time $\tau$ in an orientation-dependent manner (64, 65, 66, 67). As shown in (68) and discussed in (69), a simpler explanation can be found by coupling a theory of MIPS to a logistic growth law. The latter describes the tendency of bacterial populations to move towards a stable 'target' density at which cell death and cell division are in balance. If this target density lies within the two-phase region of the MIPS, then coexisting uniform phases are unstable to population change, whereas a uniform state at the target density is unstable to phase separation. The result is a kind of micro-phase separation which leads to patterns similar to those observed experimentally in growing colonies (68).

\subsection{Experiments on Synthetic Swimmers}

A variety of self-phoretic colloid particles undergo self-propulsion, in the presence of a fuel supply such as dissolved hydrogen peroxide $(70,71)$ or another source of stored internal energy (72). In most cases the observed system is quasi-two dimensional, because such swimmers tend to accumulate at container walls. (Also most of the swimming mechanisms used would not be sustainable for long in $d=3$ without running out of fuel (73).) Such studies often report clustering $(5,6,7)$, perhaps caused in part by attractive interactions. Similar clusters are seen in bacterial systems with colloidal attractions induced by polymer (74); in the self-phoretic context the attractions could instead arise kinetically through cross-particle responses to reagent and product gradients $(5,7,75)$. At higher densities, bulk phase separation has been reported, and attributed to a MIPS-like mechanism (6).

Even if attractive interactions are also present, it seems plausible that MIPS-related physics is implicated in the observation of stable cluster phases $(5,7,74)$. In these cases, partial phase separation occurs but seemingly gets arrested after the formation of clumps of modest size. Such clusters can be interpreted in kinetic terms as the consequence of the mutual stalling of two particles in head-on collision, which then present an obstacle that causes other particles to stall when they hit it (34). But this is also the kinetic interpretation of the MIPS mechanism: as such, it is far from obvious why this process should ever be self-limiting. (Recall that in passive phase separation, without long-range repulsive interactions, two small phase-separated droplets can always lower their interfacial energy by merging.) One idea is that weak activity could oppose, rather than enhance, a passive tendency to phase separate, creating motion that will break droplets apart (76). Indeed, this effect is already seen in purely repulsive ABPs, and is one of the reasons why very large system sizes are needed to reliably distinguish bulk phase separation from steady state density fluctuations at intermediate length scales (55).

In summary, there is some evidence for MIPS in synthetic colloidal swimmers, although much more would be welcome. In any case there is an unsolved mystery concerning the apparently widespread formation of finite clusters that, unlike their counterparts in passive systems, fail to achieve full phase separation.

One possible explanation for the absence of fully-fledged MIPS in ABP experiments involves hydrodynamic interactions. At low densities, these cause flocking and structure 
formation $(77,78)$, but at high density hydrodynamic interactions tend to suppress MIPS $(79,80)$. This has been explained as follows. To model collisional slowing down with a theory in which $v$ depends on a coarse-grained density $\rho$, one arguably needs particles to undergo several collisions before changing direction, so that the density is sampled, and an average $v$ can be defined, at scales below $\ell$. This ceases to hold at high density, because hydrodynamic torques cause particles to undergo a large rotation each time they meet (79). Though certainly important in $d=2(79,80)$, the strength of this effect in $d=3$ is so far unknown. In three dimensions then, hydrodynamic interactions could be responsible for the arrest of coarsening or, if we are unlucky, they could destroy MIPS entirely.

\section{BEYOND THE LOCAL APPROXIMATION}

In this section we review more recent work that takes the theoretical picture of MIPS beyond the local approximation. This step is essential to fully understand the dynamics of phase separation, which is driven, in passive systems, by interfacial tension. As mentioned already, within the local approximation there are no gradient terms in the free energy functional, (given by Eq. 26 and Eq. 27) so that the interfacial tension of the equivalent passive system is strictly zero (50). Hence the dynamics of MIPS entirely depends on terms so far neglected.

\subsection{Phenomenological Approach}

A natural phenomenological ansatz, which is also suggested by some types of simplified kinetic theory (48), is to add a square gradient term with constant coefficient $\kappa$ to the free-energy functional of the equivalent passive system:

$$
\mathcal{F}=\int\left[f(\rho)+\frac{\kappa}{2}(\nabla \rho)^{2}\right] d^{d} \mathbf{r}
$$

where $f(\rho)$ obeys Eq. 27. Hence

$$
\mu=\delta \mathcal{F} / \delta \rho=\ln \rho+\ln v(\rho)-\kappa \nabla^{2} \rho
$$

This maintains the mapping between MIPS and passive phase separation (37), and leads to the well known Cahn-Hilliard-Cook equation (50):

$$
\begin{aligned}
& \dot{\rho}=-\nabla . \mathbf{J} \\
& \mathbf{J}=-M \nabla \mu+\sqrt{2 M} \boldsymbol{\Lambda}
\end{aligned}
$$

where $M=D \rho$ is called the collective diffusivity. This approach has the great benefit of simplicity. In combination with a logistic population growth, it was used successfully in (68) to address patterning in bacterial colonies. It also allows a large body of knowledge on the passive case, for instance concerning critical behavior, to be adopted en masse.

\subsection{Continuum Model: Nonintegrable Gradient Terms}

A more general study requires a systematic gradient expansion of which the local approximation is the zeroth order term. In this context there is no reason to expect the corrections to obey the integrability condition, Eq. 23, as was noted in (37). Accordingly one should expect the mapping between MIPS and passive phase separation to break down at this 
level. The simplest approach (55) is to ignore any gradient contributions arising from the nonlocality of $M(\mathbf{r})=D([\rho], \mathbf{r}) \rho$ within Eq. 44, but study systematically those arising from the nonequilibrium chemical potential, $\mu=\mu_{\mathrm{id}}+\mu_{\mathrm{ex}}$. Here $\mu_{\mathrm{ex}}$ is still defined via Eq. 23 as $\mu_{\text {ex }}(\mathbf{r}) \equiv \ln v([\rho], \mathbf{r})$ but this can no longer in general be written as a functional derivative $\delta \mathcal{F}_{\text {ex }} / \delta \rho$. In contrast to this, a nonlocal diffusivity $M$ does not destroy integrability and, unless something else does, has little effect on kinetics (with a few exceptions (81)).

Focusing therefore on $\mu_{\mathrm{ex}}$, the approach of (55) is to assume

$$
\mu_{\mathrm{ex}}(\mathbf{r})=\ln v(\hat{\rho}(\mathbf{r}))
$$

where $\hat{\rho}(\mathbf{r})$ is a smeared density found by convolution of $\rho$ with an isotropic local kernel whose range is comparable to the persistence length $\ell=v(\rho) \tau$. This is the length scale on which one particle samples the density of its neighbors before changing orientation. Importantly, this range is itself density-dependent, at least for ABPs on which we focus here. When $\rho$ is slowly varying we have $\hat{\rho}=\rho+\gamma^{2} \nabla^{2} \rho$ with $\gamma=\gamma_{0} v(\rho) \tau$ and $\gamma_{0}$ of order unity. Further expanding Eq. 45 in gradients then gives

$$
\mu=\ln \rho+\ln v(\rho)-\kappa(\rho) \nabla^{2} \rho
$$

where $\kappa(\rho)=-\gamma_{0}^{2} \tau^{2} v(\rho) v^{\prime}(\rho)$. Since $\kappa$ is not constant, this form of $\mu$ is nonintegrable. One can however define a 'nearest integrable model' as

$$
\mathcal{F}=\int\left[f(\rho)+\frac{\kappa(\rho)}{2}(\nabla \rho)^{2}\right] d^{d} \mathbf{r}
$$

for which the chemical potential instead reads

$$
\mu_{D B}=\ln \rho+\ln v(\rho)-\kappa(\rho) \nabla^{2} \rho-\frac{\kappa^{\prime}(\rho)}{2}(\nabla \rho)^{2}
$$

The last term is an inevitable partner to a density-dependent $\kappa$ coefficient in any system that obeys the principle of detailed balance. Its absence for MIPS has interesting consequences $(55,61)$ that we described in the next section.

Equations 43, 44 and 46 comprise an explicit continuum model for MIPS whose only apparent input (modulo the order unity factor $\gamma_{0}$ ) is the chosen function $v(\rho)$. For ABPs, this is available from Eq. 39 but, as previously mentioned, a correction term must also be added to $f(\rho)$ to prevent the density surpassing the close-packed limit, $\rho^{*}$. The resulting theory can be compared with both direct ABP simulations and the 'nearest integrable model' (comprising Eqs. 43, 44 and 48). Figure 7 shows $L(t)$ curves for all three cases in $d=2,3$, with apparent scaling exponents in $d=2$ somewhat below the value of $1 / 3$ expected for diffusive coarsening in passive systems $(55,56)$. However, this shift is seen for the nearest integrable model, as well as for the active continuum model with DB violations (55). Suggestively, passive coarsening is known to show an altered exponent in the case where $M$ vanishes in the dense phase (81), and so could give misleading corrections to the asymptotic scaling when, as in ABPs, the diffusivity is very small there.

More generally, the continuum model using Eq. 46 gives a reasonably good account of domain shapes and dynamics when compared with direct ABP simulations. However, local fluctuations that violate time-reversal symmetry are under-represented in the continuum model $(55,56,57)$, for reasons that are not yet understood. Such fluctuations are prohibited altogether in the 'nearest integrable model'. The fact that this prohibition has little effect on $L(t)$ shows these DB-violating fluctuations to be subdominant, at least in determining the rate of domain growth. 

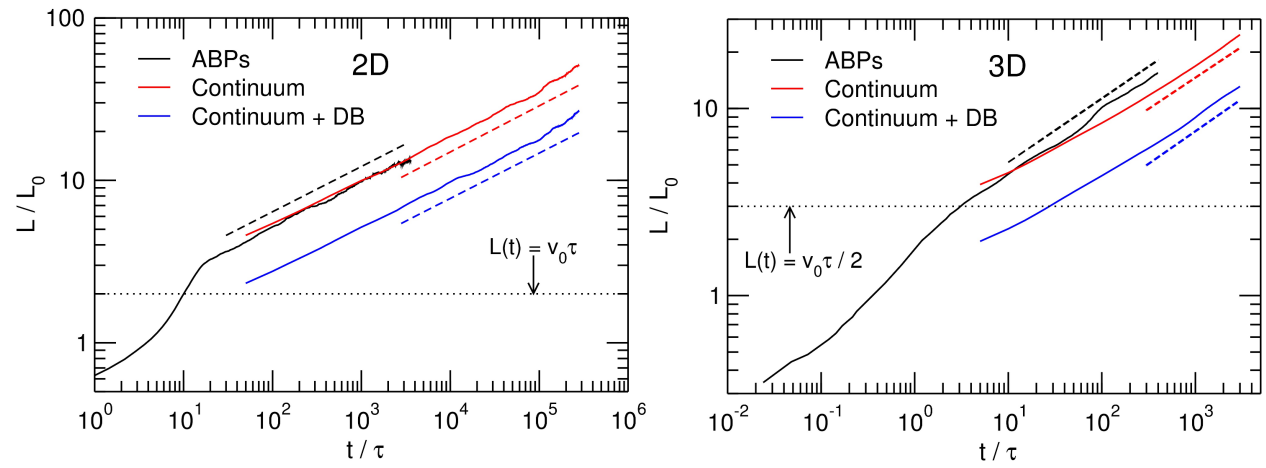

Figure 7

(Left) Coarsening curves for ABPs, the continuum model violating detailed balance, and (shifted downwards for clarity) the nearest integrable model in $d=2$. (Right) the same in $d=3$. Dashed lines correspond to exponent $\simeq 0.28$ in $d=2$ and $\simeq 0.33$ in $d=3$. Adapted from (56), courtesy of J. Stenhammar.

\subsection{Anomalous Phase Behavior: Active Model B}

A surprising numerical observation made in (55) is that the non-integrable gradient terms, while they have little consequence for coarsening dynamics, do affect the densities $\left(\rho_{1}, \rho_{2}\right)$ of the coexisting phases. At first sight this is odd, because the common tangent construction makes no mention of any gradient terms. However, it does implicitly assume thermodynamic equilibrium and hence DB. There are other instances in physics where nonintegrable gradient terms alter an equilibrium result that appears not to involve gradients $(82,83)$.

The corresponding implications for MIPS were explored in (61) using a non-integrable generalization of 'Model B'. The latter is a canonical model for diffusive phase separation in passive systems (84). It introduces an order parameter $\phi$ which is, in this section, not the volume fraction but a linear transform of the particle density $\rho$ chosen so that the coexistence densities are at $\phi_{1,2}= \pm 1$. Model B then writes a free energy functional

$$
\mathcal{F}=\int\left[-\frac{\phi^{2}}{2}+\frac{\phi^{4}}{4}+\frac{\kappa}{2}(\nabla \phi)^{2}\right] d^{d} \mathbf{r}
$$

with constant $\kappa$. The chemical potential is

$$
\mu=\delta \mathcal{F} / \delta \phi=-\phi+\phi^{3}-\nabla^{2} \phi
$$

where we have set $\kappa=1$ without loss of generality (this amounts to a rescaling of length). Suppressing any $\phi$-dependence of $M$ and choosing time units so that $M=1$ gives

$$
\dot{\phi}=-\nabla^{2} \mu+\nabla \cdot \Lambda
$$

Eq. 50 and Eq. 51 comprise Model B for the purposes of passive phase separation studies. The model gives $L(t) \sim t^{1 / 3}$, and captures other dynamical features such as the nucleation and growth kinetics in the regions of positive $f^{\prime \prime}$ within the common-tangent binodals.

Active Model B (61) replaces Eq. 50 with

$$
\mu=-\phi+\phi^{3}-\nabla^{2} \phi+\lambda(\nabla \phi)^{2}
$$



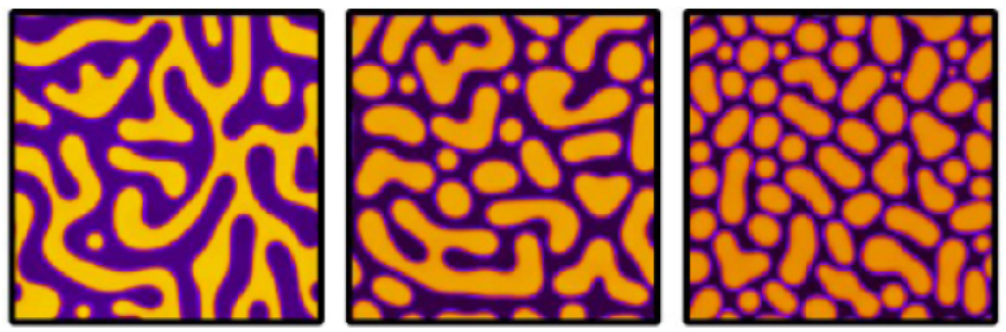

Figure 8

Domain morphology in Active Model B during coarsening in $d=2$, for $\phi=\phi_{0}=0$ and $\lambda=0,-1,-2$ (left, centre and right respectively). Courtesy of R. Wittkowski; adapted from (61).

where the $\lambda$ term renders the model non-integrable, and can be viewed as the 'distance' to the nearest integrable model (namely, Passive Model B). This structure can be compared with Eq. 46 and Eq. 48 for ABPs. In the latter case, $\kappa(\rho)$ depends on density but a term in $\kappa^{\prime}(\rho)(\nabla \rho)^{2}$ is missing from $\mu$. In Active Model $\mathrm{B}, \kappa=1$ is constant but a term in $(\nabla \phi)^{2}$ is added. For simplicity this has a constant coefficient $\lambda$ which can have either sign but is negative for ABPs (61). What matters is the mismatch between the $\nabla^{2} \phi$ and $(\nabla \phi)^{2}$ terms in the expression for $\mu$; Active Model B captures this in the simplest possible way.

The model is simple enough to make analytical progress (61). One finds that the common tangent construction is replaced by an 'uncommon tangent' in which the two coexisting phases have the same chemical potential $\mu$ but unequal values of $\phi \mu-f$ which is, in thermodynamic language, the pressure. The reason for a shift in the coexistence conditions is, in this language, a discontinuity in pressure across the interface. Note that $\nabla \phi$ is not negligible in the interfacial region; indeed it is responsible (via $\kappa$ ) for the interfacial tension. The $\lambda$ term supplements this by a jump in thermodynamic pressure, which is linear in $\lambda$ for small values, but saturates at large ones in such a way that the coexisting densities can approach, but not enter, the spinodal region (61). This kind of behavior is not possible in equilibrium, and shows that the DB violations that underly MIPS cannot be transformed away entirely. Nonetheless the corrections are weaker than they might have been. For instance, once DB is violated there is no guarantee that the densities of coexisting phases stay constant when the overall mean density in the system is changed. However, the uncommon tangent construction does preserve this feature.

Active Model B explains the deviation from the common-tangent construction that was seen for ABP simulations (55). However, a careful study of its coarsening behavior gives predictions qualitatively unaltered from the passive case, although numerically there is once again evidence of a reduced exponent for the temporal scaling of the domain size $L(t)$. Figure 8 shows snapshots for various $\lambda$ taken during the coarsening process. Notably, Active Model B does not exhibit saturation of $L(t)$ at length scales smaller than the system size, and therefore cannot explain the existence of cluster phases in which coarsening has been reported to arrest at an intermediate length scale $(5,6,7,74)$.

A tempting way forward from Active Model B is to construct an Active Model $\mathrm{H}$, in which the $\phi$ field is coupled to a momentum-conserving solvent. For passive systems, the path from Model B to Model $\mathrm{H}$ is clear (84), but this involves a thermodynamic relationship connecting the mechanical stress to a free energy derivative which breaks down for active matter. At the time of writing, Active Model H therefore remains under development (61). 


\section{CONCLUSION}

In this review we have focused on one specific aspect of active matter physics: the ability of motile particles, with isotropic interactions whose only or main effect is to slow their propulsion speed at high density, to undergo phase separation. By ignoring the complexities presented by many real types of active matter, particularly orientational interactions, workers on this topic have been able to create a fairly detailed theoretical and numerical understanding of the underlying physics. Large parts of this can be understood in terms of an equivalent system of passive particles with attractive interactions. This is interesting since it allows a highly developed area of near-equilibrium statistical mechanics to be deployed in modeling this specific class of active systems.

However, the equivalence is not complete, and its breakdown is also interesting. One finds by simulation that, although much of the behavior familiar in passive phase separation is retained, some aspects of motility-induced phase separation (MIPS) irreducibly violate detailed balance at mesoscopic and even macroscopic scales. Indeed, during phase separation, on a mesoscopic scale within the growing domains, particle currents arise that plainly violate time reversal symmetry on that length scale. For active Brownian particles these include lava-lamp type motion, and/or bubbles of the minority phase forming continuously within a domain and then moving to its surface $(56,57)$. Macroscopically, one observes modest but clear deviations in the densities of coexisting phases from those predicted by globally minimizing an effective free energy. These features can be reproduced in part by simplified continuum models in which detailed balance is violated by gradient terms only.

Possibly because they address only 'active simple fluids' (without orientational interactions) the connection between theories of MIPS reviewed here and experiment remain somewhat tenuous at present. However, phenomena resembling phase separation are certainly seen in some types of experiments on bacteria and on synthetic colloidal swimmers. In the latter case, there seems to be a generic tendency to form 'cluster phases' in which phase separation arrests at a finite domain size. It is not yet clear whether this is a modified form of MIPS (perhaps with passive attractions causing the arrest) or a modified form of passive, attraction-driven phase separation (perhaps arrested by activity).

This conundrum highlights a current deficiency of the theory: so far we do not have a framework to combine MIPS-type effective attractions with standard (i.e., passive) colloidal interactions inside a single set of equations. The same basic obstacle arises whenever MIPSlike physics is coupled to other phenomena: orientational interactions, mixtures of active and passive particles, and hydrodynamic forces. Such couplings are also very challenging to address numerically, but one or more of them is present in most experimental cases. In the absence of decisive experimental evidence for MIPS despite extensive numerical evidence, it may yet turn out that, as far as experimental systems of ABPs are concerned, MIPS is the dog that did not bark. ${ }^{4}$ Its basic mechanism is extremely simple: a positive feedback between slowing-induced accumulation and accumulation-induced slowing. When both features are present, absence of MIPS may itself impart useful mechanistic information about what else is happening in the system. For example, as mentioned in Sec. 6.2, hydrodynamic interactions could suppress MIPS by causing inter-particle torques (79).

\footnotetext{
"Inspector Gregory: "Is there any other point to which you would wish to draw my attention?" Holmes: "To the curious incident of the dog in the night-time."

Gregory: "The dog did nothing in the night-time."

Holmes: "That was the curious incident." (85)
} 
Interaction torques are of course also important, even without hydrodynamic interactions, for aspherical swimmers. The full physics of orientational interactions in active rods requires one to address non-scalar order parameters describing either polar (vector) or nematic (tensor) order. Theories of orientational ordering and its effects in active systems have been extensively developed in recent years as reviewed in $(12,86)$, both for 'dry' systems (without momentum conservation, such as vibrated granular rods) and 'wet' ones such as bacterial swarms in solution. Even without condensation into an orientationally ordered state, incipient rotational order can enhance the tendency to undergo MIPS in systems where a density-dependent swim speed $v(\rho)$ is combined with an additive translational diffusion $D_{t}(87)$. Furthermore, when MIPS induces the formation of dense droplets, aligning interactions may lead to the appearance of local order, hence making the droplets self-propel (88). All this should be relevant to the study of active Brownian rods or dimers $(20,74,89,90,91,92,93)$ where MIPS-like phenomena, such as aggregation and swarm formation have been reported. Since interparticle torques convey vectorial information between particles, this is an area where the physics of the Vicsek model (9) (which give flocking via alignment interactions at fixed $v$ ) and that of MIPS (which stems solely from slowing of $v$ at high density) overlap. This could be a fruitful area for future studies.

Finally, we mention the literature on simulating discrete non-spherical swimmers with hydrodynamic interactions $(94,95,96)$. While some of the observed phenomena may well be related to MIPS, the additional presence of both near-field alignment interactions and farfield hydrodynamics, makes any connection difficult to establish without further research.

\section{ACKNOWLEDGMENTS}

The authors thank particularly Joakim Stenhammar, and also Rosalind Allen, Richard Blythe, Fred Farrell, Christina Marchetti, Davide Marenduzzo, Ignacio Pagonabarraga, Wilson Poon, Adriano Tiribocchi, Alasdair Thompson, Raphael Wittkowski, and Chantal Valeriani, for discussions and collaborations relevant to this article. MEC is funded by a Royal Society Research Professorship and EPSRC Grant EP/J007404. This research was supported in part by the National Science Foundation under Grant No. NSF PHY11-25915.

\section{LITERATURE CITED}

1. Ballerini M, Cabibbo N, Candelier R, Cavagna A, Cisbani E et al., 2008. Proc. Natl. Acad. Sci. USA 105, 1232-37.

2. Katz Y, Tunstrøm K, Ioannou CC, Huepe C, Couzin ID. 2011, Proc. Natl. Acad. Sci. USA 108, 18720-25.

3. Schaller V, Weber C, Semmrich C, Frey E, Bausch AR, 2010. Nature 467 73-77.

4. Sumino Y, Nagai KH, Shitaka Y, Tanaka D, Yoshikawa K et al., 2012. Nature 483 448-52.

5. Theurkauff I, Cottin-Bizonne C, Palacci J, Ybert C, Bocquet L, 2012. Phys. Rev. Lett. 108, 268303.

6. Buttinoni I, Bialke J, Kummel F, Lowen H, Bechinger C, Speck T. 2013. Phys. Rev. Lett. 110, 238301.

7. Palacci J, Sacanna S, Steinberg AP, Pine DJ, Chaikin PM, 2013. Science 339, 936-40.

8. Bricard A, Caussin JB, Desreumaux N, Dauchot O, Bartolo D, 2013. Nature 503, 95-98.

9. Vicsek T, Czirók A, Ben-Jacob E, Cohen I, Shochet O, 1995. Phys. Rev. Lett. 75, 1226.

10. Romanczuk P, Bär M, Ebeling W, Lindner B, Schimansky-Geier L, 2012. Europ. Phys. Jour. Special Topics 202, 1-162. 
11. Cates ME, 2012. Repts. Prog. Phys. 75, 042601.

12. Marchetti MC, Joanny JF, Ramaswamy S, Liverpool TB, Prost J, Rao M, Simha RA, 2013. Rev. Mod. Phys. 85, 1143.

13. Flemming HC, 2002. App. Microbiol. and Biotech. 59, 629-640.

14. Costerton JW, Montanaro L, Arciola CR, 2005. Int. J. Artificial Organs 28, 1062-1068.

15. Hall-Stoodley L, Costerton JW and Stoodley P, 2004. Nat. Rev. Microbiol. 2, 95-108.

16. Lee KY, Mooney DJ, 2001. Chem. Rev. 101 1869-1879.

17. Logan BE, Regan JM, 2006. Trends. Microbiol. 14 512-518.

18. Sokolov A, Aranson IS, Kessler JO and Goldstein RE, 2007. Phys. Rev. Lett. 98158102.

19. Ramaswamy S, Simha RA, Toner J, 2003. EPL 62, 196.

20. Peruani F, Deutsch A and Baer M, 2006. Phys. Rev. E 74, 030904(R).

21. Solon A, Tailleur J, 2013. Phys. Rev. Lett. 111, 078101.

22. Cisneros LH, Cortez R, Dombrowski C, Goldstein RE, Kessler JO, 2007. Expts. in Fluids 43 737-53.

23. Tailleur J, Cates ME, 2009. EPL 86, 600002.

24. Palacci J, Cottin-Bizonne C, Ybert C, Bocquet L. 2010, Phys. Rev. Lett. 105, 088304.

25. Enculescu M, Stark H, 2011. Phys. Rev. Lett. 107, 058301.

26. Galajda P, Keymer J, Chaikin P, Austin R, 2007. J. Bacteriol. 1891033.

27. Wan MB, Reichhardt CO, Nussinov Z, Reichhardt C, 2008. Phys. Rev. Lett. 101018102.

28. Di Leonardo R, Angelani L, Dell'Arciprete D, Ruocco G, Iebba V et al, 2010. Proc. Nat. Acad. Sci. USA 107 9541-45.

29. Angelani L, Di Leonardo R and Giancarlo R, 2009. Phys. Rev. Lett. 102048104.

30. Sokolov A, Apodaca MM, Grzybowski BA, Aronson IS, 2010. Proc. Nat. Acad. Sci. USA 107 969-974.

31. Miller MB, Bassler BL, 2001. Ann. Rev. Microbiol. 55 165-199.

32. Thompson AG, Tailleur J, Cates ME, Blythe RA, 2011. J. Stat. Mech. P02029.

33. Fily Y, Marchetti MC, 2012. Phys. Rev. Lett. 108, 235702.

34. Redner GS, Hagan MF, Baskaran A, 2013. Phys. Rev. Lett. 110, 055701.

35. Bialké J, Löwen H, Speck T, 2013. EPL 103, 30008.

36. Levis D, Berthier L, 2014. arxiv:1403.3410.

37. Tailleur J, Cates ME, 2008. Phys. Rev. Lett. 100, 218103.

38. Schnitzer MJ, 1993. Phys. Rev. E 48, 2553-68.

39. van Kampen NG, 1988. J. Phys. Chem. Solids 49, 673-77.

40. Schnitzer MJ, Block SM, Berg HC, Purcell EM, 1990. Symp. Soc. Gen. Microbiol. 46, 15-33.

41. Berg HC, 2003. E. coli in Motion (Springer, New York)

42. Krapivsky PL, Redner S, Ben-Naim E, 2010. A Kinetic View of Statistical Physics. (Cambridge University Press).

43. Cates ME, Tailleur J, 2013. EPL 101, 20010.

44. Dean DS, 1996. J. Phys. A 29, L613-17

45. Øksendal B, 2003. Stochastic Differential Equations: an Introduction with Applications. (Springer Berlin Heidelberg).

46. Liu C, Fu X, Liu L, Ren X, Chau CK, Li S et al., 2011. Science 334, 238-241.

47. Fu X, Tang LH, Liu C, Huang JD, Hwa T et al., 2012. Phys. Rev. Lett. 108, 198102.

48. Speck T, Bialké J, Menzel AM, Löwen H. 2013. arXiv:1312.7242.

49. Onuki A, 2002. Phase Transition Dynamics (Cambridge University Press).

50. Bray AJ, 2002. Adv. Phys. 51, 481.

51. Fily Y, Henkes S, Marchetti MC, 2014. Soft Matter 10, 2132-40.

52. Schtz G, Sandow S, 1994. Phys. Rev. E 49, 2726.

53. Tailleur J, Kurchan J, Lecomte V, 2008. J. Phys. A 41, 505001.

54. Soto R, Golestanian R, 2014. Phys. Rev. E 89, 012706.

55. Stenhammar J, Tiribocchi A, Allen RJ, Marenduzzo D, Cates ME, 2013. Phys. Rev. Lett.111, 
145702 .

56. Stenhammar J, Marenduzzo D, Allen RJ, Cates ME, 2014. Soft Matter 14, 1489-99.

57. Wysocki A, Winkler RG, Gompper G, 2014. EPL 105, 48004.

58. Berthier L, 2013. arXiv:1307.0704.

59. Henkes S, Fily Y, Marchetti MC, 2011. Phys. Rev. E 84, 040301.

60. Redner GS, Baskaran A, Hagan MF, 2013. Phys. Rev. E 88, 012305.

61. Wittkowski R, Tiribocchi A, Stenhammar J, Allen RJ, Marenduzzo D, Cates ME, 2013. arXiv:1311.1256.

62. Nash RW, Adhikari R, Tailleur J, Cates ME, 2010. Phys. Rev. Lett. 104, 258101.

63. Sutherland IW, 2001. Microbiol. UK 147, 3-9.

64. Budrene EO, Berg HC, 1991. Nature 349, 630-3.

65. Woodward DE, Tyson R, Myerscough MR, Murray JD, Budrene EO, Berg HC, 1995. Biophys. J. 68, 2181-89.

66. Tyson R, Lubkin SR, Murray JD, 1999. Proc. R. Soc. Lond. Ser. B 266, 299-304.

67. Budrene EO, Berg HC, 1995. Nature 376, 49-53.

68. Cates ME, Marenduzzo D, Pagonabarraga I, Tailleur J, 2010. Proc. Nat. Acad. Sci. USA 107, 11715-20.

69. Brenner MP, 2010. Proc. Nat. Acad. Sci. USA 107 11653-65.

70. Howse JR, Jones RAL, Ryan AJ, Gough T, Vafabakhsh R, Golestanian R, 2007. Phys. Rev. Lett. 99, 048102.

71. Ibele M, Mallouk TE, Sen A, 2009. Ang. Chem. Int. Edn. 48 3308-12.

72. Thutupalli S, Seemann R, Herminghaus S, 2011. New J. Phys. 13, 073021; Dreyfus R et al. 2005. Nature 437 862-865.

73. Brown A, Poon WCK, 2014. Soft Matter 10, 4016-4027.

74. Schwarz-Linek J, Valeriani C, Cacciuto A, Cates ME, Marenduzzo D et al., 2012. Proc. Nat. Acad. Sci. USA 109 4052-4057.

75. Saha S, Golestanian R, Ramaswamy S, 2013. arXiv: 1309.4947

76. Mognetti BM, Sarić A, Angioletti-Uberti S, Cacciuto A, Valeriani C, Frenkel D, 2013. Phys. Rev. Lett. 111, 245702.

77. Ishikawa T, Pedley TJ, 2008. Phys. Rev. Lett. 100, 088103.

78. Llopis I, Pagonabarraga I, 2006. EPL 75, 999-1005.

79. Fielding SM, 2012. arXiv:1210.5464.

80. Pohl O, Stark H, 2014. arXiv:1403.4063.

81. Bray AJ, Emmott CL, 1995. Phys. Rev. B 52, R685-R688.

82. Lu CYD, Olmsted PD, Ball RC, 2000. Phys. Rev. Lett. 84 642-645.

83. Watson SJ, Norris SA, 2006. Phys. Rev. Lett. 96, 176103.

84. Chaikin P, Lubensky TC, 1995. Principles of Condensed Matter Physics. (Cambridge University Press.)

85. Conan-Doyle, A., 1894. The Memoirs of Sherlock Holmes. Ch.1: Silver Blaze. (George Newnes Ltd., London.)

86. Ramaswamy S, 2010. Ann. Rev. Cond. Mat. Phys. 1 323-245.

87. Barre J, Chetrite R, Muratori M, Peruani F, 2014. arXiv:1403.2364

88. Farrell FDC, Marchetti MC, Marenduzzo D, Tailleur J. 2012. Phys. Rev. Lett. 108, 248101.

89. Baskaran A, Marchetti MC, 2008. Phys. Rev. Lett. 101268101.

90. Ginelli F, Peruani F, Baer M, Chate H, 2010. Phys. Rev. Lett. 104184502.

91. Wensink H, Löwen H, 2012. J. Phys. Cond. Mat. 24464130.

92. McCandlish SR, Baskaran A, Hagan MF, 2012. Soft Matt. 8 2527-34.

93. Abkenar M, Marx K, Auth T, Gompper G, 2013. Phys. Rev. E 88062314.

94. Hernandez-Ortiz JP, Graham MD, 2005. Phys. Rev. Lett 95204501.

95. Saintillan D, Shelley MJ, 2007. Phys. Rev. Lett 99058102.

96. Saintillan D, Shelley MJ, 2008. Phys. Rev. Lett 100178103. 